\title{
Digestion-Ligation-Only Hi-C, a simple, cost-effective, and highly efficient method for chromosome conformation capture
}

\author{
D. Lin ${ }^{1 \#}$, P. Hong ${ }^{2 \#}$, G. Li ${ }^{2 *}$, G. Cao ${ }^{1 *}$ \\ ${ }^{1}$ State Key Laboratory of Agricultural Microbiology, Huazhong Agricultural University, Wuhan, China \\ ${ }^{2}$ National Key Laboratory of Crop Genetic Improvement, Huazhong Agricultural University, Wuhan, \\ China \\ *e-mail: guoliang.li@mail.hzau.edu.cn (G.L.),gcao@mail.hzau.edu.cn (G.C.)
}

Key words: genomics, sequencing technology, chromosome conformation, Hi-C

Chromosome conformation capture technologies open an avenue to investigate the three-dimensional (3D) structures of genomes. However, high noise, high cost, and lack of straightforward noise evaluation in current methods impede the advancement of 3D genomic research. Here, we developed a simple Digestion-Ligation-Only Hi-C (DLO $\mathrm{Hi}-\mathrm{C})$ technology to explore the 3D landscape of the genome. This method requires only two rounds of digestion and ligation without biotin-labeling and pull-down for reducing the cost. The noise DNA was efficiently removed in a cost-effective step by purifying specific linker-ligated DNA fragments. Notably, random ligation could be quickly evaluated in an early quality-control step before sequencing. Moreover, we performed an in situ version of DLO Hi-C method based on 4-cutter restriction enzyme. We applied DLO Hi-C to delineate the genomic architecture of THP-1 and K562 cells and uncovered chromosome translocations. This technology may facilitate the investigation of genomic organization, gene regulation, and (meta-) genome assembly.

\# These authors contributed equally to this work. 